\section{Interactions between Parasite and Host in Human Visceral Leishmaniasis}

\author{
Harizanov*, Rumen $\mathbf{N}$ and Kaftandjiev Iskren $\mathbf{T}$ \\ Department of Parasitology and Tropical Medicine, National Center of \\ Infectious and Parasitic Diseases, Sofia, Bulgaria
}

protozoa. Subsequently, Ronald Ross, using Leishmans staining technique named these protozoan microorganisms "Leishman bodies", and Manson named them "Leishman-Donovan bodies"-in memory of the two English scientists. The name, "kala-azar" (black disease) was given by McNaught, who described the disease in India. Swaminath et al., using volunteers proved that the disease is transmitted to humans by insects and found those specific biological carriers are bloodsucking insects from genus Phlebotomus. For first time Rogers was able to cultivate the parasite in citrated blood, but his attempts to maintain the strain by passaging were unsuccessful. Later, Ch. Nicolle was able to cultivate and maintain Leishmania strain by passaging and also to induce experimental disease in dogs and monkeys. He first highlights the epidemiological link between man and $\operatorname{dog}[1,2]$.

\section{Etiology}

\section{Taxonomy}

Genus Leishmania, depending on which part of the alimentary canal of the insect vector carries out its development is divided into two subgenera-Leishmania and Viannia. Promastigotes relating to subgenera Leishmania, realize part of their biological cycle in front and middle part of the alimentary canal (suprapilar development) of the specific biological vector and those of subgenera Viannia, in the rear part (peripilar development) [3,4]. The species belonging to subgenera Leishmania, are divided into three groups: L. donovani comlex ( $L$. donovani, $L$. infantum and $L$. chagasi), who are viscerotropic and cause visceral leishmaniasis; $L$. tropica complex (L. tropica, L. aethiopica, L. major), which are dermatotropic and cause cutaneous leishmaniasis; L. mexicana complex (L. mexicana, L. amazonensis, L. pifanoi, L. garhami, L. venezuelensis)-causative agents of mucocutaneous leishmaniasis [3]. Subgenera Viannia is found only in the New World and includes various species belonging to $L$. brasiliensis complex (L. guyanensis, $L$. naiffi, L. peruviana, L. panamensis, L. shawi) [5].

Genus Leishmania belongs systematically to Kingdom Protista; Subkingdom Protozoa; Family Tripanosomatidae [6]. Taxonomic identification of the different species Leishmania is based on two types of features-external (clinical manifestations, geographical distribution, epidemiological cycle) and internal (morphological and molecular structure) [4,7].

Parasite classification at genus level has been based on global taxonomics derived in the 1990s using isoenzyme technique in comparison with reference strains. Identification depends on the employed method, e.g. zymodemes (parasite populations with common isoenzyme patterns identified electrophoretically) or schizodemes (parasite populations defined by shared 'fingerprint patterns' obtained by a process involving digestion of kinetoplast DNA by restriction enzymes). The results are relevant in descriptive epidemiology and allows grouping of the parasites into hierarchies that suggest their evolutionary relations [8].

In the Mediterranean basin, $L$ infantum displays a broad enzymatic polymorphism with 20 different zymodemes, of which 18 have been found in humans. L. infantum MON-1 is the most frequent 
zymodeme in humans and is usually responsible for VL along the Mediterranean, region more rarely for CL [9].

\section{Morphology and life cycle}

In the biological development of Leishmania spp. there are two distinct morphological stages: amastigote stage (also called leishmanial stage) in the final host-human or animal, and promastigote (infective stage, flagellate stage, leptomonade stage) in the specific carrier-bloodsucking insects of genus Phlebotomus (Old World) and family Lutzomia (New World) $[8,10,11]$. The amastigotes (Figure 1) are small, oval, intracellular bodies, measuring 2 to $5 \mu \mathrm{m}$ long and 1 to $3 \mu \mathrm{m}$ wide, with a large nucleus and kinetoplast (rod) arranged against it and are covered by a densely packed glycocalyx primarily composed of glycoinositolphospholipids and glycosphingolipids [12]. Amastigotes are found in the vacuoles of the phagocytic cells of the mononuclear phagocyte system (macrophages, monocytes, histiocytes, endothelial cells), where they multiply by simple binary fission to produce 20-30 new leishmanial amastigotes until the host cell is destroyed and the released amastigotes invade new cells. Through macrophages the parasite reaches all organs and tissues of the host organism $[8,10,13]$.

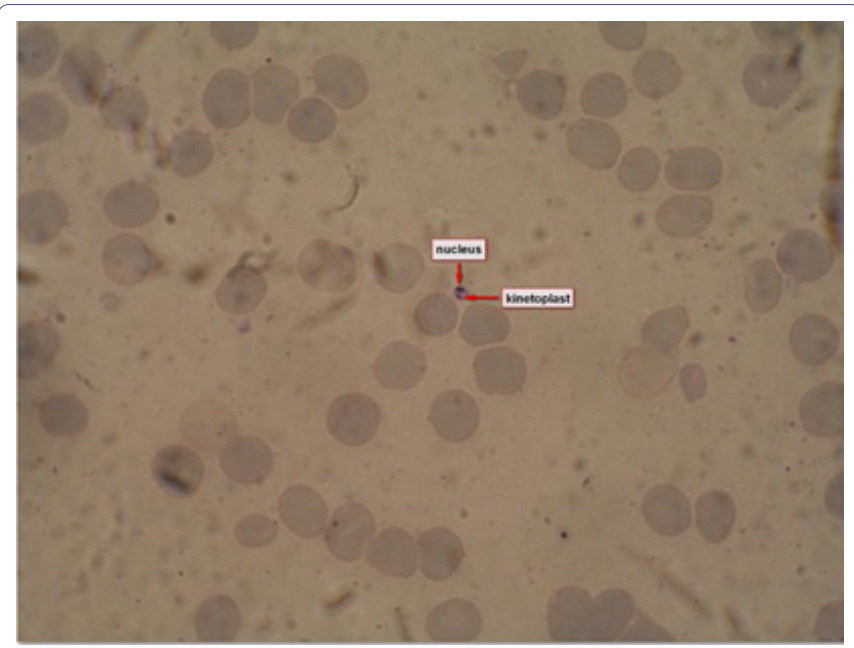

Figure 1: Leishmanial amastigote in bone marrow specimen from a patient with visceral leishmaniasis.

Note: Red arrows indicate the nucleus and kinetoplast of the parasite.

When taking a blood meal from human or animal carrier the female sand fly ingest with the blood also hosts cells invaded by amastigotes. Then the amastigotes are released into the midgut of the digestive tract of the insect where are transformed into elongated flagellate promastigotes (procyclic promastigotes) and in a few days later their number increases significantly. The promastigotes migrate to the front section of the digestive tract where are transformed into short, spherical, non-dividing promastigotes (metacyclic promastigotes) and when the phlebotomine sand fly takes another blood meal it will inoculate 10 to 200 promastigotes (Figure 2) in the dermis of a vertebrate host $[8,10,11]$. From there they fall into the phagocytic cells of the immune system, including macrophages, dendritic cells and neutrophils where will transform again to amastigotes and begin cycle of reproduction [14]. The infected macrophages spread from the dermis to other tissues and organs such as spleen, liver and bone marrow. Different species Leishmania possess specific tropism, and some of them are dermatotropic (causing cutaneous and mucocutaneous leishmaniasis), while others are viscerotropic (causing visceral leishmaniasis). The reasons for existence of specific tropism are not clear yet, but it is assumed that depends on some genetic features of the host and the parasite, as well as some external factors such as temperature. For example dermatotropic Leishmania spp. can not realize their biological cycle at the ambient temperature of the internal organs of a vertebrate host as are able the viscerotropic Leishmania spp. [15-19].

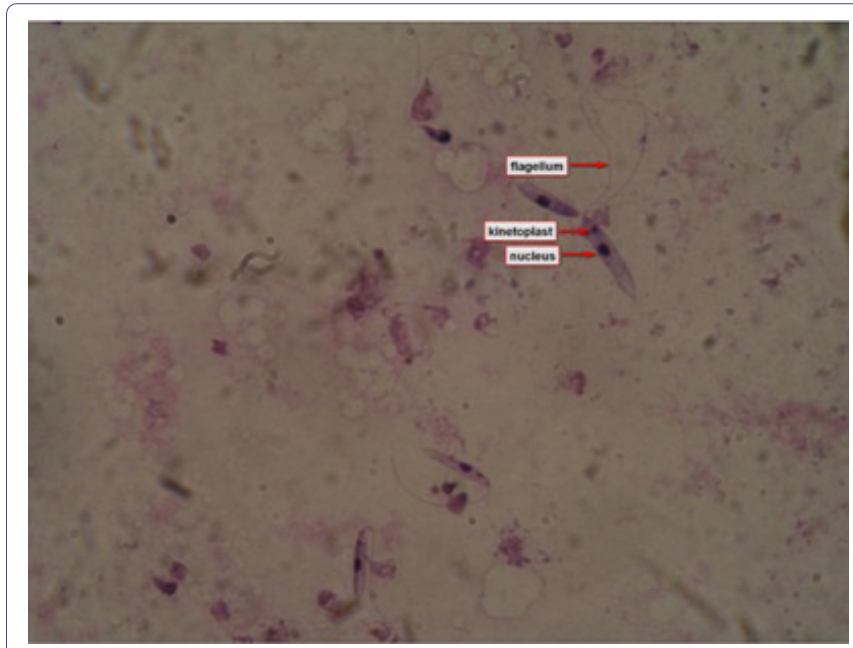

Figure 2: Leishmanial promastigotes from culture in triple $\mathrm{N}$ (NNN-Novy, MacNeal, Nicolle) medium.

Note: Red arrows indicate the nucleus, kinetoplast and flagellum of the motile form.

\section{Epidemiology}

Clinical features of visceral leishmaniasis show some differences between endemic, epidemic and sporadic cases. In most cases, the disease is a zoonosis with a source and reservoir of wild rodents or representatives of family Canidae, but with ability to infect humans $[4,8,20]$. Risk factors for the disease are malnutrition, immunosuppressive therapy, and AIDS [21-25]. According to WHO susceptibility of people to visceral leishmaniasis is the same with exception of those who have acquired immunity after previous disease $[4,8]$. Existing data suggest a slight prevalence of incidence among males as males/females ratio is $4: 3$. The intensity of transmission of the disease is directly related to the environmental conditions and consequently the circumstances, under which a person is infected, vary significantly in time and place. This is especially true in cases of visceral leishmaniasis of zoonotic type where the epizootic process takes place outside and independently of man in sylvatic foci, and man is infected only accidentally if his activities (work, hunting, hiking, wars, etc.) get him exposed to the sylvatic transmission cycle. Dogs are main reservoir of infection in synanthropic foci, and the presence of pets additionally attracts phlebotomine sand flies. In such areas, the households are in fact micro-foci for transmission of visceral leishmaniasis $[4,8,26]$.

\section{Geographic distribution}

Visceral leishmaniasis is widespread all over the world:

In the Mediterranean Basin-visceral leishmaniasis is the main form of the disease. It occurs in rural areas, in villages in mountainous regions and also in some periurban areas, where reservoir hosts for the parasite are dogs and other animals $[4,8,27]$. 
In South-East Asia visceral leishmaniasis is the main form of the disease. Transmission most often occurs in rural areas below $600 \mathrm{~m}$ above sea level, with a heavy annual rainfall, mean humidity above $70 \%$, a temperature range of $15-38^{\circ} \mathrm{C}$, abundant vegetation, subsoil water and alluvial soil. The disease is most common in agricultural villages where houses are frequently constructed with mud walls and earthen floors, and livestock is living close to humans [4,8,27].

In East Africa there are frequent outbreaks of visceral leishmaniasis in in the northern Acacia-Balanite savanna and the southern savanna and forest areas where sandflies live around termite mounds [4,8,27].

Americas-visceral leishmaniasis is very similar to that found in the Mediterranean Basin [4,8,27].

The disease is highly endemic in the Indian subcontinent and in East Africa. An estimated 200, 000 to 400, 000 new cases of VL occur worldwide each year. Over $90 \%$ of new cases occur in six countries: Bangladesh, Brazil, Ethiopia, India, South Sudan, and Sudan [27].

\section{Pathogenesis and Pathology}

\section{Pathogenesis and histological changes in individuals with uncompromised immunity}

During a blood meal phlebotomine sand fly takes about 0,5 $\mu$ l. blood per meal (quantity much less than the one which can be transmitted using the same syringe) and regurgitates the parasite after that. It has been found that when the parasite density in sand fly is high, the large number of leishmania in the proboscis hinders the process of feeding [28-32]. For that reason the vector penetrates multiple times the skin of the host, as a result of which are carried out multiple inoculations of the parasite. Together with leishmanial promastigotes within the host are passed also peptide inhibitors of macrophages and vasodilators. Studies have shown that these vasodilators, e.g. maksidilan (isolated from L. Longipalpis), enables even inoculation of a minimum number of parasites to be infective, and also causes a long-lasting erythema at the bite site. While un-infective procyclic promastigotes are sensitive to complement-mediated cytolysis, the infective metacyclic promastigotes carried by the vector are relatively resistant $[4,8,10,16,20,24]$.

Macrophages, the primary target of intracellular Leishmania infection, may take on distinct phenotypes in response to parasite signals and inflammatory stimuli within the infected microenvironment. Classically activated (M1) macrophages respond to IFN $\gamma$ and microbial products by generating antimicrobial molecules that effectively kill Leishmania and other intracellular pathogens. Central to the killing of intracellular parasites is the production of nitric oxide by the action of inducible nitric oxide synthase 2 on the substrate L-arginine. In contrast, alternatively activated or M2 macrophages, which are typically generated by exposure to type 2 cytokines (IL-4, IL-13), fail to produce antimicrobial effector molecules to kill intracellular pathogens and serve to dampen inflammation and promote wound healing [33,34]. However, as other infectious agents, most Leishmania species have evolved effective strategies to evade the innate immune response during the early moments of infection, and this is by rapidly blocking the induction and regulation of key host cell functions including production of nitric oxide, tumour necrosis factor-alpha, interleukin-12, and radical oxygen species [35]. Peripheral blood mononuclear cells from patients with manifested VL typically do not proliferate or produce IFN $\gamma$ in response to Leishmanial Antigen (LAg).
A few months after completion of therapy, following cure, proliferative and cytokine responses to LAg are usually detectable [36]. Leishmania parasites have been shown to alter host-cell signaling pathways to promote their survival. Activation of host Src homology 2 domain containing tyrosine phosphatases by Leishmania infection results in global dephosphorylation of tyrosine residues, leading to deactivation of a variety of signaling pathways including JAK/STAT, NF- $\mathrm{kB}$, IRF-1, and MAP kinases. Increased concentrations of secondary messengers including calcium $\left(\mathrm{Ca}^{2+}\right)$, inositol lipids, inositol phosphatases, and protein kinase $\mathrm{C}$ have also been observed following Leishmania infection. Induction of the negative regulatory proteins, suppressors of cytokine signaling, have been characterized following Leishmania infection and interfere with cytokine signaling and host cell activation [12].

On the sites of inoculations are formed small granulomas (leishmaniomas), which consists of histiocytes containing parasites. They are surrounded by epithelioid cells, which are gradually transformed into giant cells. Subsequent development depends on the host immune response, ranging from missing to a response that leads to a full eradication of the parasite. From the site of inoculation, and after transformation into amastigotes the leishmania parasites localize in the regional lymph nodes, from where they pass into the blood stream and through it to other cells of the reticuloendothelial system of the end host. It develops hyperplasia of the reticuloendothelial cells of the liver, spleen, bone marrow, lymph nodes, lining of the small intestine, and other lymphoid tissues. Increase of the spleen size is due to reticuloendothelial proliferation and, in some cases can be so extreme that its caudal part may reach the iliac fossa. The spleen is with dense consistency and full with congested blood. In the acute stage of the disease its capsule is smooth, within the pulp are observed multiple infarct areas and massive intracellular invasion of the histiocytes by amastigotes. Histologic studies of liver changes in visceral leishmaniasis showed that the Kupffer cells in the portal areas contain numerous parasites $[4,8,10,16,20,24]$. In most cases, efficient immune responses to L. donovani in the liver depend on the formation of granulomas, a process influenced by chemokine production, subsequent recruitment of monocytes, neutrophils, CD4+ T cells and CD8+ T cells, production of inflammatory cytokines and activation of infected cells [37]. Lymph nodes may be enlarged and contain histiocytes infected with amastigotes. Bone marrow is poor in cellular elements as myelocytes and promyelocytes predominate. At the beginning hematopoiesis is normal, but in the course of illness the life of erythrocites and leukocytes shortens and that leads to anemia and granulocytopenia with relative lymphoid and monocytosis. Subsequently is reduced the synthesis of prothrombin. This in combination with growing thrombocytopenia can lead to severe bleeding from mucous membranes. The course of the disease may be complicated with diarrhea, ulceration of the intestinal mucosa, and enteritis in untreated cases. In the late stage may develop secondary infections such as pneumonia and tuberculosis, which often lead to death. Activation of the complement system may enhance developing anemia due to circulating immune complexes, but complications such as development of glomerulonephritis have been reported rarely. Reticuloendothelial massive proliferation induces enlargement of the liver and spleen, which upon proper treatment may restore their original size $[4,8,20,24,38,39]$. 


\section{Pathogenesis in leishmania/HIV co-infection}

In leishmania/HIV co-infection the suppression of cellular immunity is more pronounced. This is due to a complex of cytokines who inhibit the production of interferon which reduces macrophages lytic abilities. The symptoms of HIV-infection dominate these of leishmaniasis. Immunosuppression caused by HIV does not allow for adequate cell mediated immune response against the parasite. The reduction of $\mathrm{CD} 4+\mathrm{T}$-cells leads to a lack of T-antigen recognizing cells and consequent failure of B-lymphocytes stimulation. This leads to an oligoclonal B cells response, which explains the increased incidence of false negative serological tests in co-infected persons. HIV-mediated inhibition of the proliferative immune response to the parasite creates conditions for its dissemination and atypical location in host tissues and organs. In other hand leishmanial invasion facilitates replication of HIV by causing chronic immune activation, one of the main conditions for development of AIDS [24,40,41]. Coinfection with HIV reduces in-vitro cellular and Th1 cytokine responses to Leishmania and increases Th2 cytokine responses. The Leishmania promastigote and its lipophosphoglycan induce HIV-1 replication, which correlates with CD4+ T-cell loss by apoptosis. If the clinical response to antimonials is good, HIV replication decreases-if it is poor, HIV replication increases [42]. Immune activation facilitates the stimulation of viral coreceptors (CCR5 and CXCR4), reduces the secretion of cytokines, promotes the entry of the virus into the host cells and helps for subsequent disintegration of $\mathrm{T}$ cells. It also leads to an increased secretion of TNF- $\alpha$, IL-2, IL-4, IL-6, IL-10 and altered cellular metabolism. Co-infection causes immune dysfunction in several levels and reduced efficiency of the immune response and cell death, which leads to progression of immune deficiency and a reduced survival of patients $[24,40,41]$. In contrast from patients with HIV-monoinfection, in co-infected is found significant increase of the receptors for IL-4, IL-10, IL-2, and reduction of post-stimulative production of IFN- $\gamma$. It has been found that lipophosphoglycanes of L. infantum can induce expression of HIV-1 in latently infected peripheral blood mononuclear cells, possibly via secretion of TNF- $\alpha$. The effect of visceral leishmaniasis on HIV-infection is detected after it has been observed simultaneous increase of the amount of HIV-1 RNA with that of IL-4, IL-6 and IL-10, TNF- $\alpha$. On the other hand, successful treatment of HIV-associated visceral leishmaniasis results in reduced levels of TNF- $\alpha$ and plasma viremia [43].

\section{Immunity}

Studies by Dominguez and Torano showed that after entry of the parasite in the host organism leishmania promastigotes bind with natural IgM antibodies in 30 seconds, which leads to activation of the complement system in the classical way [40]. In it, the first component $\mathrm{C} 1$ of the cascade is connected with the antigen-antibody complex but not all antibodies have the ability to activate $\mathrm{C} 1$. Such abilities have the antibodies from classes IgG and IgM, while those from classes $\operatorname{IgE}, \operatorname{IgD}$ and $\operatorname{Ig} \mathrm{A}$ do not. Even in some cases $\operatorname{IgA}$ can inhibit the complement activation from IgG. As a result of the activation of the classical complement cascade, the parasite is opsonized with participation of the third component of the Cascade [C3]. Opsonised promastigotes bind to erythrocytic CR1-receptors. Course of infection continues by transfer of opsonized promastigotes from erythrocytes to monocytes and macrophages, using CR3-receptors. Since the activation does not lead to CR3 activation of oxidative processes in the phagocytic cells, it appears that the parasite uses the components of the complement system, to facilitate its smooth penetration into the cells of the reticuloendothelial system
$[20,40,44,45]$. Besides the classical pathway is activated also the alternative pathway of the complement system in which proteins S3b S3bi bind with larger molecules from the cell membrane of promastigotes-gp63 and Lipophosphoglycane (LPG). After getting into in the phagolysosomes of macrophages, promastigote forms are transformed into amastigotes. Begins multiplication by binary fission, resulting in rupture of the invaded cells and invasion of new cells $[40,44,46]$. Another common mechanism also plays role in the immunity against Leishmania.The infection stimulates monocytes, marcrophages and primary Dendritic Cells (DCs) which associate with the TSC-mTOR pathway to manipulate the inflammatory response by balancing the IL-10 and IL-12. IL-12 is proinflammatory factor while IL-10 is anti-inflammatory factor in which the homeostasis of two factors impose substantial protection to the host. Disrupted balance would result in fatal damage to the hosts cells, one of the examples is cytokine storm. Infections would initiate the change of the axis between IL-10 and IL-12, in specific, inhibition of mTOR would escalate the IL-12 in Leishmania donovani infected THP-1-derived macrophages via PI3K/Akt pathway. IL-10 seems to be independent to inhibition of PI3K pathway but depends on inhibition of mTOR. The mediation is also related to TLR2, where blocking the TLR2 leads to suppression of IL-10 expression [47]. It is also proposed that Leishmania can evade macrophages antimicrobial actions by decreasing the activity of transcription factors such as Activated Protein-1(AP-1) that are involved in transcription of genes coding for antimicrobial functions of macrophages via its surface protein GP63 that inactivates AP-1 transcription factor. GP63 enter into the macrophages independently of parasite internalization using lipid rich microdomains localized in the cellular membrane. Then GP63 was observed to reaches the nuclear compartment and cleaves AP-1 subunit proteins [48].

\section{T-cells immune response in visceral leishmaniasis}

As part of the immune response of the host against Leishmania for susceptibility to invasion, help the T-helper cell type 2 (Th2), and for resistance to it, those of type Th1, and this is because Th2 secrete IL-4, IL- 5 and IL-10, while Th 1 secrete IL- 2 and IFN- $\gamma$. It has been shown that IFN- $\gamma$ activates iNOS2 enzyme (macrophage nitrous oxide synthetase), that stimulates the output of NO (nitrogen monoxide), which kills the intracellular amastigotes. The host susceptibility to invasion is due to the production of IL- 4 and IL- 10 by Th 2 , which blocks the production of IFN- $\gamma$ from Th 1 and thus a parasiticide function of macrophages and enables the parasite to realize, unhindered the intracellular development. As typical parasites leishmania ensure its development by modulating the host immune response-causing immunosuppression or stimulate functions favorable for their development $[40,49,50]$. In response to infection is reduced the production of interleukin- 1 and -2 , interferon-gamma and tumor necrosis factor alpha (TNF- $\alpha$ ) by the T cells, who are recovering after treatment. Suppression of cell-mediated immunity leads to uncontrolled reproduction and dissemination of parasites $[40,44,45]$. In addition, IL-12 is absent in Leishmania antigen-induced cytokine production in peripheral blood mononuclear culture supernatants, and IL-2 and IFN- $\gamma$ are either absent or present in low levels1. These findings reinforce the view that it is mainly the Th1 response that is suppressed [51].

\section{B-cells immune response in visceral leishmaniasis}

It is known that in vivo B cellular immune response is generally ineffective due to the intracellular localization of the parasite. In 

001 .

classical and severe disease usually the level of specific anti-leishmanial antibodies is high. In such cases develops a non-specific polyclonal B-cell activation, leading to hypergammaglobulinemia [40]. In VL usually is observed increased production of globulins, mainly at the expense of classes of IgG and IgM.

Target of the humoral immune response are some heat shock proteins (Hsp). Antibodies against Hsp 70 have been detected in $92 \%$ of patients with VL [44]. In blood sera of the patients can be found circulating immune complexes with immunoglobulins of classes A, G and M. Skin patch delayed hypersensitivity reaction is suppressed or even absent. After recovery this sensitivity is restored [40,52].

\section{Clinical Presentation and Complications}

Visceral leishmaniasis is a kind of reticuloendotheliosis and is directly related to the immune status of infected individuals. Clinical manifestations of VL can range from asymptomatic subclinical carrier to manifested, severe forms of the disease. After incubation period ranging from 10 days to more than a year (mean of 2 and 4 months) appear the first symptoms of the disease. Depending on the species of viscerotropic leishmaniae the beginning may be indolent or noticeable-protracted or severe. In the first case the symptoms can be vague, with a sense of discomfort, while acute onset disease resembles typhoid fever or malaria. Described are several clinical forms of VL [24].

\section{Light subclinical (oligosymptomatic) form of VL}

In this clinical version of visceral leishmaniasis, the clinical picture is poor and it is difficult to direct the therapist to etiological diagnosis if not taking into consideration the epidemiological history-travel or stay in an endemic area. Usually the leading symptom is general weakness, most often accompanied by gastro-intestinal manifestations and in some cases protracted fever. For example, after the operation "Desert Storm" none of about 500000 participating American troops fell down ill with classic symptoms of VL, but 9 were diagnosed with oligosimptomatic form [53]. The patients complained of chronic fatigue, nausea, cough, intermittent diarrhea, or abdominal pain and fever. The clinical manifestations in these patients were similar to cases of VL as described earlier in children in Brazil and were defined by the authors as "subclinical" form of the disease [54-56]. These data gave reason to a number of authors to consider that in both developed and developing countries the most common form of visceral leishmaniasis is the oligosymptomatic. Due to this fact any patient with generalized symptoms and with epidemiological data for residing or traveling in regions endemic for the disease needs to undergo a parasitological examination $[24,56]$.

\section{Classical form of VL}

After incubation period ranging from 10-15 days to 10 months or more develop the general symptoms of the disease. In this clinical form the symptoms include fever, enlargement of the spleen and liver (splenohepatomegaliy), reduction in the number of erythrocytes, leukocytes and platelets to complete pancytopenia and weight loss leading to cachexia. In the course of the disease can be distinguished three stages-initial, stage of splenohepatomegaly and stage of cachexia.

Initial stage: The earliest clinical symptom is development of primary lesion-ulcer at the site of inoculation, which can be detected in the prodromal period prior general clinical manifestations to appear.
Typically, in the initial stage the first manifestation of VL is increased body temperature, which is constant or remitting, and later can become intermittent with two or three peaks a day [20,24,57-60].

Stage of splenohepatomegaly: Gradually, amid prolonged febrile condition develops enlargement of the spleen and liver, lymphadenopathy, accompanied in some cases by acute abdominal pain. Darkening of the skin of the face, hands, feet and abdomen is observed in the Indian version of VL (kala-azar). Clinical examination reveals that splenic enlargement is more pronounced than hepatomegaly. The spleen is usually palpable at about $5-15 \mathrm{~cm}$ below the left rib arc. According to some authors, splenomegaly may be absent in about $5 \%$ of the cases [61]. Anemia, weight loss and pronounced splenohepatomegaliy occur with disease progression. Anemia is a symptom, almost always accompanying visceral leishmaniasis, and in some cases may be of high grade. It is usually normocytic and normochromic, and is caused by various factors, including bone marrow damage by the parasites, signs of hypersplenism, haemorrhage and hemolysis. Leukopenia is common, and in some cases on this background develop secondary infections. Thrombocytopenia may lead to bleeding (haemorrhagic) diathesis $[57,58]$. In some cases of VL can be found hypergammaglobulinemia, circulating immune complexes and positive rheumatoid factor. Seldom, the circulating immune complexes can cause development of glomerulonephritis in the kidneys [62]. Also have been described cases of combination of VL with acute hepatitis [63], bacteremia [64], Guillain-Barre syndrome [65]. Malabsorption is a significant problem in children with VL and is a consequence of adverse effects of the cell-mediated immunity [66]. In cases of disease in India and Sudan have been observed Post-kala-azar Dermal Leishmaniasis (PKDL). These nodular or papular skin lesions contain large number of amastigotes and are of great epidemiological importance for the spread of VL.

Stage of cachexia: Usually develops because of delayed diagnosis and/or improper treatment. In patients in this stage is observed a significant reduction of body weight. This is accompanied by aggravation of the haematological changes with development of hemorrhagic diathesis. Without treatment, the clinically manifested VL is usually fatal $[8,20,57,58,67]$.

\section{Sudanese clinical variation of VL (Sudanese disease; Killing disease)}

Visceral leishmaniasis is endemic in the territory of Sudan, where is established high prevalence of about $4 \%$. Besides the classic manifestations of clinical symptoms it also includes some unusual symptoms and complications such as burning pain in the extremities, combined with other neurological symptoms, leishmanial cholecystitis, colitis and many cases of congenital visceral leishmaniasis. An interesting feature is the development of PKDL in about $56 \%$ of cases. In these cases the disease course is identical with the Indian clinical and epidemiological version of visceral leishmaniasis. In perioral areas develops maculopapular rash. In cases when there is no spontaneous recovery, lesions spread to other parts of the face, in the upper part of the chest, hands or genitals, as some of them may develop ulcerations. There is a significant difference: while in India PKDL develops months to years after treatment in Sudanese patients skin lesions occur in the range 0 to 180 days (mean 56 days) [24,68-70]. According to some authors Sudanese visceral leishmaniasis is a separate clinical and epidemiological version of visceral leishmaniasis, because they are differences compared with the 

001 .

Indian and Mediterranean version both in terms of clinical symptoms and in some epidemiological features. For example, it has been found that source and reservoir of the infection might be some rodents such as rats or squirrels [10].

\section{VL associated with AIDS}

In Southern Europe and other geographic areas endemic for visceral leishmaniasis in recent years there is an increase in the cases of coinfection. While in Europe in 1994 were recorded 300 cases of co-infection, by 1997 their total number reached about 1000 cases, and is established parity between the cases leishmania/HIV co-infection and those with visceral leishmaniasis alone [24]. Clinical manifestations of VL in HIV-infected persons are very similar with the classic form of the disease [71,72]. The incubation period is variable, some authors consider that it is related to the age of the diseased [7]. Most infected are males (80-85\%) and clinical symptoms include fever, hepatomegaly and/or splenomegaly, hypergammaglobulinemia, and pancytopenia [7,71]. In about 20 to $40 \%$ of patients hepatosplenomegaly is absent and the level of CD4 cells is low $(<50$ CD4 cells/mm) [71-76]. In some cases of VL/HIV there are present detectable specific immune antibodies, while in other there are not. Besides the classic manifestations of the disease in cases of coinfection often can be observed involvement of the gastrointestinal tract [stomach, duodenum, or colon]; complications like ascites; pleural or pericardial effusion; involvement of the lungs, tonsils, skin, and even simultaneous impairment of all organs and systems of the body $[22,76]$. Anemia is caused by a number of factors and is associated with the antiretroviral therapy, autoimmune hemolysis, splenic enlargement and loss of blood from the gastrointestinal tract. The blood loss is increased in thrombocytopenia. Agranulocytosis and superinfection occur in untreated patients. In approximately $70 \%$ of coinfected patients is observed asthenia, anorexia, weight loss, and in about $60 \%$ there is lymphadenopathy [7]. Cases of visceral leishmaniasis with similar symptomatology may be observed also in immunocompetent persons receiving immunosuppressive therapy [24].

\section{Diagnosis and Treatment options}

The gold standard for diagnosis of all leishmaniases is demonstration of parasite (amastigotes) in a tissue biopsy. Serological tests, such as ELISA, DAT, IFA and rk39 test, are indicative of VL in combination with clinical symptoms, but can not reliably differentiate between past and recent infections. In recent years, have been developed bio-molecular methods (PCR) for diagnosis and species determination of the causative agent of the disease $[36,67]$.

In most cases visceral leishmaniasis is curable disease. Several treatment options are available but their usage and efficacy depend on a variety of factors. These factors include parasite species, severity of the symptoms and the immune status of the host. The only treatment currently available for visceral leishmaniasis relies on chemotherapy $[12,77]$. Classical, first-line drug treatments include pentavalent antimonials compounds, stibogluconate (Pentostam) or meglumine antimonite (Glucantime). Pentavalent antimonials have been in use for more than 50 years and are the recommended treatment by the World Health Organization (WHO) $[4,8]$. Other common drugs include amphoterecin B, lipid formulations of amphoterecin B, including liposomal amphotericin B, amphotericin B lipid complex and amphotericin B colloidal dispersion, pentamidine, miltefosine (Impavido or Miltex) and imidazoles, allopurinol (Zyloric), or paromomycin among others.
However, several obstacles remain in the treatment of visceral leishmaniasis Most treatments require extensive (weeks to months) and invasive (intramuscular or intravenous) modes of administration and there is a high rate of significant drug-related toxic side effects. The cost of treatment is often well beyond the means of many patients and severe toxicity may require secondary treatment, adding to the already high cost of therapy $[12,69]$.

\section{Conclusion}

Host-parasite interactions are many and various in nature. In the review we have tried to present briefly some interactions between the agents of visceral leishmaniasis and hosts cells. Their further study could lead to improved diagnostic methods and development of new therapeutic formulations and vaccines which to introduce in the clinical practice.

\section{References}

1. Thakur $\mathrm{CP}$, History of Kala-azar.

2. Gibson ME (1983) The identification of kala-azar and the discovery of Leishmania donovani. Med Hist 27: 203-213.

3. Lainson R, Shaw JJ (1987) Evolution, classification and geographical distribution. The Leishmaniasis in Biology and Medicine 1: 1-120.

4. Control of the leishmaniasis (1990) WHO, Technical Report Series. Geneva, Switzerland.

5. Shaw JJ (1994) Taxonomy of the genus Leishmania: present and future trends and their implications. Mem Inst Oswaldo Cruz 89: 471-478.

6. Golemanski V, Schischinyova M (2001) Zoology of Invertebrates. Gera Art Sofia.

7. Evans TG (1993) Leishmaniasis. Infect Dis Clin North Am 7: 527-546.

8. Control of the leishmaniasis (2010) Report of a meeting of the WHO Expert Committee on the Control of Leishmaniases, Geneva.

9. Sulahian A, Garin YJF, Pratlong F, Dedet JP, Derouin F (1997) Experimental pathogenicity of viscerotropic and dermotropic isolates of Leishmania infantum from immunocompromised and immunocompetent patients in a murine model. FEMS Immunology and Medical Microbiology 17: 131-138.

10. Neva FA, Brown HW (1994) Basic clinical parasitology. (6 $6^{\text {th }}$ edn). Prentice-Hall International Inc.

11. Hommel M (1999) Visceral leishmaniasis: Biology of the parasite. J Infect 39: $101-111$.

12. Boggiatto $P(2010)$ Leishmaniasis: immunologic indicators of clinical progression and mechanisms of immune modulation. Graduate Theses and Dissertations. lowa State University Paper.

13. Bogitsh BJ, Carter CE, Oeltmann TN (2005) Human Parasitology. (3rdedn). Elsevier Academic Press.

14. Sacks D, Sher A (2002) Evasion of innate immunity by parasitic protozoa. Nat Immunol 3: 1041-1047.

15. McCall LI, Zhang WW, Matlashewski G (2013) Determinants for the development of visceral leishmaniasis disease. PLoS Pathog 9:1003053.

16. Scott $P$ (1985) Impaired macrophage leishmanicidal activity at cutaneous temperature. Parasite Immunol 7: 277-288.

17. Wittner M, Tanowitz HB (2000) Leishmaniasis in infants and children. Semin Pediatr Infect Dis 11: 196-201.

18. Callahan HL, Portal IF, Bensinger SJ, Grogl M (1996) Leishmania spp: temperature sensitivity of promastigotes in vitro as a model for tropism in vivo. Exp Parasitol 84: 400-409. 
Citation: Harizanov, Rumen N, Kaftandjiev Iskren T (2014) Interactions between Parasite and Host in Human Visceral Leishmaniasis. J Cytol Tissue Biol 1: 001 .

19. McCall LI, Matlashewski G (2010) Localization and induction of the A2 virulence factor in Leishmania: evidence that $\mathrm{A} 2$ is a stress response protein. Mol Microbiol 77: 518-530.

20. Garcia LS, Buckner DA (1993) Diagnostic Medical Parasitology. (2ndedn) American Society for Microbiology, Washington, USA.

21. Seaman J, Mercer AJ, Sondorp E (1996) The epidemic of visceral leishmaniasis in western Upper Nile, southern Sudan: course and impact from 1984 to 1994. Int J Epidemiol 25: 862-871.

22. Alvar J, Cañavate C, Gutiérrez-Solar B, Jiménez M, Laguna F, et al. (1997) Leishmania and human immunodeficiency virus coinfection: the first 10 years. Clin Microbiol Rev 10: 298-319.

23. Desjeux P (1996) Leishmaniasis. Public health aspects and control. Clin Dermatol 14: 417-423

24. Berman JD (1997) Human leishmaniasis: clinical, diagnostic, and chemotherapeutic developments in the last 10 years. Clin Infect Dis 24: 684-703.

25. Guerin PJ, Olliaro P, Sundar S, Boelaert M, Croft SL, et al. (2002) Visceral leishmaniasis: current status of control, diagnosis, and treatment, and a proposed research and development agenda. Lancet Infect Dis 2: 494-501.

26. Harizanov R, Rainova I, Tzvetkova N, Kaftandjiev I, Bikov I, et al. (2013) Geographical distribution and epidemiological characteristics of visceral leishmaniasis in Bulgaria, 1988 to 2012. Euro Surveill 18: 20531.

27. Leishmaniasis (2014) WHO, Fact sheet $\mathrm{N}^{\circ} 375$.

28. Jefferies D, Livesey JL, Molyneux DH (1986) Fluid mechanics of bloodmeal uptake by Leishmania-infected sandflies. Acta Trop 43: 43-53.

29. Killick-Kendrick R (1986) The transmission of leishmaniasis by the bite of the sandfly J Roy Army Med Corps 132: 134-140.

30. Warburg A, Schlein Y (1986) The effect of post-bloodmeal nutrition of Phlebotomus papatasi on the transmission of Leishmania major. Am J Trop Med Hyg 35: 926-930.

31. Schlein Y, Jacobson RL, Messer G (1992) Leishmania infections damage the feeding mechanism of the sandfly vector and implement parasite transmis sion by bite. Proc Natl Acad Sci USA 89: 9944-9948.

32. Bates PA (2007) Transmission of Leishmania metacyclic promastigotes by phlebotomine sand flies. Int J Parasitol 37: 1097-1106.

33. Martinez FO, Helming L, Gordon S (2009) Alternative activation of macrophages: an immunologic functional perspective. Annu Rev Immunol 27: 451483.

34. Osorio EY, Travi BL, da Cruz AM, Saldarriaga OA, Medina AA, et al. (2014) Growth Factor and Th2 Cytokine Signaling Pathways Converge at STAT6 to Promote arginase expression in progressive experimental visceral leishmaniasis. PLoS Pathog 10: 1004165.

35. Olivier M, Atayde VD, Isnard A, Hassani K, Shio MT (2012) Leishmania virulence factors: focus on the metalloprotease GP63. Microbes Infect 14: 1377 1389.

36. Kumar R, Nylén S (2012) Immunobiology of visceral leishmaniasis. Front Immunol 3: 251.

37. Stanley AC, Engwerda CR (2007) Balancing immunity and pathology in visceral leishmaniasis. Immunol Cell Biol 85: 138-147.

38. Gutierrez Y, Maksem JA, Reiner NE (1984) Pathologic changes in murine leishmaniasis (Leishmania donovani) with special reference to the dynamics of granuloma formation in the liver. Am J Pathol 114: 222-230.

39. Giunchetti RC, Mayrink W, Carneiro CM, Corrêa-Oliveira R, Martins-Filho OA, et al. (2008) Histopathological and immunohistochemical investigations of the hepatic compartment associated with parasitism and serum biochemical changes in canine visceral leishmaniasis. Res Vet Sci 84: 269-277.

40. Melhorn H (2004) Encyclopedic Reference of Parasitology. (2ndedn). Springer-Verlag Berlin Heidelberg, Newyork.
41. Murray HW, Berman JD, Davies CR, Saravia NG (2005) Advances in leishmaniasis. Lancet 366: 1561-1577.

42. Report of the Fifth Consultative Meeting on Leishmania/HIV Coinfection (2007) WHO, Addis Ababa, Ethiopia.

43. Paredes R, Munoz J, Diaz I, Domingo P, Gurgui M, et al. (2003) Leishmaniasis in HIV infection. J Postgrad Med 49: 39-49.

44. Wallace GR, Ball AE, MacFarlane J, el Safi SH, Miles MA, et al. (1992) Mapping of a visceral leishmaniasis-specific immunodominant B-cell epitope of Leishmania donovani Hsp70. Infect Immun 60: 2688-2693.

45. Galvão-Castro B, Sá Ferreira JA, Marzochi KF, Marzochi MC, Coutinho SG et al. (1984) Polyclonal B cell activation, circulating immune complexes and autoimmunity in human american visceral leishmaniasis. Clin Exp Immunol 56: 58-66.

46. Sacks D, Sher A (2002) Evasion of innate immunity by parasitic protozoa. Nat Immunol 3: 1041-1047.

47. Tse MT, Kwan P (2013) mTOR and its Physiological Impacts-Part II: Immunological Impact. Journal of Biochemical and Pharmacological Research 1:138-142.

48. Contreras I, Gómez MA, Nguyen O, Shio MT, McMaster RW, et al. (2010) Leishmania-induced inactivation of the macrophage transcription factor AP-1 is mediated by the parasite metalloprotease GP63. PLoS Pathog 6: 1001148

49. Awasthi A, Mathur RK, Saha B (2004) Immune response to Leishmania infection. Indian J Med Res 119: 238-258.

50. Sundar S, Reed SG, Sharma S, Mehrotra A, Murray HW (1997) Circulating T helper 1 (Th1) cell- and Th2 cell-associated cytokines in Indian patients with visceral leishmaniasis. Am J Trop Med Hyg 56: 522-525.

51. Goto H, Prianti Md (2009) Immunoactivation and immunopathogeny during active visceral leishmaniasis. Rev Inst Med Trop Sao Paulo 51: 241-246.

52. Pearson RD, Cox G, Jeronimo SM, Castracane J, Drew JS, et al. (1992) Visceral leishmaniasis: a model for infection-induced cachexia. Am J Trop Med Hyg 47: 8-15.

53. Magill AJ, Grogl M, Johnson SC, Gasser RA Jr (1994) Visceral infection due to Leishmania tropica in a veteran of Operation Desert Storm who presented 2 years after leaving Saudi Arabia. Clin Infect Dis 19: 805-806.

54. Badaró R, Jones TC, Lorenço R, Cerf BJ, Sampaio D, et al. (1986) A prospective study of visceral leishmaniasis in an endemic area of Brazil. J Infect Dis 154: 639-649.

55. Badaro R, Jones TC, Carvalho EM, Sampaio D, Reed SG, et al. (1986) New perspectives on a subclinical form of visceral leishmaniasis. J Infect Dis 154 1003-1011.

56. Badaró R, Benson D, Eulálio MC, Freire M, Cunha S, et al. (1996) rK39: a cloned antigen of Leishmania chagasi that predicts active visceral leishmaniasis. J Infect Dis 173: 758-761.

57. Genov G (1998) Practical Parasitology, (1stedn). Knowledge, Bulgarian

58. Petrov P (1992) Parasitology (1stedn), Medicine and Physical Education, Bulgarian.

59. Conjivaram V (2013) Pediatric Leishmaniasis.

60. Harizanov RN, Kaftandjiev IT, Jordanova DP, Marinova IB, Tsvetkova ND (2013) Clinical features, diagnostic tools, and treatment regimens for viscera leishmaniasis in Bulgaria. Pathog Glob Health 107: 260-266.

61. Hashim FA, Ali MS, Satti M, el-Hassan AM, Ghalib HW, et al. (1994) An outbreak of acute kala-azar in a nomadic tribe in western Sudan: features of the disease in a previously non-immune population. Trans $\mathrm{R}$ Soc Trop Med Hyg 88: 431-432.

62. Dutra M, Martinelli R, de Carvalho EM, Rodrigues LE, Brito E, et al. (1985) Renal involvement in visceral leishmaniasis. Am J Kidney Dis 6: 22-27. 
63. Hervás JA, Albertí $P$, Ferragut J, Canet $R$ (1991) Acute hepatitis as a presenting manifestation of kala-azar. Pediatr Infect Dis J 10: 409-410.

64. Garcés JM, Tomás S, Rubiés-Prat J, Gimeno JL, Drobnic L (1990) Bacterial infection as a presenting manifestation of visceral leishmaniasis. Rev Infect Dis 12: $518-519$.

65. Fasanaro AM, Scoleri G, Pizza V, Gaeta GB, Fasanaro A (1991) Guillain-Barré syndrome as presenting manifestation of visceral leishmaniasis. Lancet 338: 1142.

66. Pearson RD, Sousa AQ (1996) Clinical spectrum of Leishmaniasis. Clin Infect Dis 22: 1-13

67. Sundar S, Rai M (2002) Laboratory diagnosis of visceral leishmaniasis. Clin Diagn Lab Immunol 9: 951-958.

68. M Nail A, M Imam A (2013) Visceral leishmaniasis: Clinical and demographic features in an African population. Pak J Med Sci 29: 485-489.

69. Chappuis F, Sundar S, Hailu A, Ghalib H, Rijal S, et al. (2007) Visceral leishmaniasis: what are the needs for diagnosis, treatment and control? Nat Rev Microbiol 5: 873-882

70. Mukhopadhyay D, Dalton JE, Kaye PM, Chatterjee M (2014) Post kala-azar dermal leishmaniasis: an unresolved mystery. Trends Parasitol 30: 65-74.
71. Montalban C, Calleja JL, Erice A, Laguna F, Clotet B, et al. (1990) Viscera leishmaniasis in patients infected with human immunodeficiency virus. Co-operative Group for the Study of Leishmaniasis in AIDS. J Infect 21: 261-270.

72. del Mar Sanz M, Rubio R, Casillas A, Guijarro C, Costa JR, et al. (1991) Visceral leishmaniasis in HIV-infected patients. AIDS 5: 1272-1274.

73. Berenguer J, Moreno S, Cercenado E, Bernaldo de Quirós JC, García de la Fuente A, et al. (1989) Visceral leishmaniasis in patients infected with Human Immunodeficiency Virus (HIV). Ann Intern Med 111: 129-132.

74. Rosenthal E, Marty P, Poizot-Martin I, Reynes J, Pratlong F, et al. (1995) Visceral leishmaniasis and HIV-1 co-infection in southern France. Trans R Soc Trop Med Hyg 89: 159-162.

75. Pintado V, Martín-Rabadán P, Rivera ML, Moreno S, Bouza E (2001) Visceral leishmaniasis in Human Immunodeficiency Virus (HIV)-infected and non-HIV-infected patients. A comparative study. Medicine (Baltimore) 80 54-73.

76. Rosenthal E, Marty P, del Giudice P, Pradier C, Ceppi C, et al. (2000) HIV and Leishmania coinfection: a review of 91 cases with focus on atypical locations of Leishmania. Clin Infect Dis 31: 1093-1095.

77. Frézard F, Demicheli C, Ribeiro RR (2009) Pentavalent antimonials: new perspectives for old drugs. Molecules 14: 2317-2336. 